\title{
Using laparoscopic ultrasound to delineate dangerous anatomy during difficult laparoscopic cholecystectomies
}

\author{
Maciej Sebastian ${ }^{A-F}$, Maciej Sroczyński ${ }^{A-F}$, Jerzy Rudnicki ${ }^{A-F}$ \\ Department of Minimally Invasive Surgery and Proctology, Wroclaw Medical University, Poland \\ A - research concept and design; $B$ - collection and/or assembly of data; $C$ - data analysis and interpretation; \\ $\mathrm{D}$ - writing the article; $\mathrm{E}$ - critical revision of the article; $\mathrm{F}$ - final approval of the article
}

Address for correspondence

Maciej Sebastian

E-mail:mseba@op.pl

Funding sources

None declared

Conflict of interest

None declared

Received on November 17, 2016

Reviewed on February 9, 2017

Accepted on August 8, 2018

Published online on June 14, 2019

Cite as

Sebastian M, Sroczyński M, Rudnicki J. Using laparoscopic ultrasound to delineate dangerous anatomy during difficult laparoscopic cholecystectomies. Adv Clin Exp Med. 2019;28(8):1037-1042. doi:10.17219/acem/94077

DOI

10.17219/acem/94077

Copyright

Copyright by Author(s)

This is an article distributed under the terms of the

Creative Commons Attribution Non-Commercial License

(http://creativecommons.org/licenses/by-nc-nd/4.0/)

\begin{abstract}
Background. Bile duct injury (BDI) during laparoscopic cholecystectomy (LC) is not as common now as in the past, but it is still a very debilitating complication. Therefore, there is a very strong need for a method that lowers the number of complications during $\mathrm{LC}$ without any additional risks for the patient and the operating team. Laparoscopic ultrasound (LUS), which serves to delineate anatomy, appears to be a very effective and safe technique.
\end{abstract}

Objectives. The aim of this study was to explore the advantages of performing LUS during difficult LC.

Material and methods. The study group consisted of 126 patients who underwent surgery between January 2014 and February 2016. All the patients had difficult intraoperative anatomical conditions due to chronic inflammation, previous upper abdominal surgery or biliary pancreatitis in the past. We used a Toshiba PEF-704 LA laparoscopic probe and the Toshiba NemioMX SSA-590A diagnostic ultrasound system (Toshiba Corp., Tokyo, Japan). Doppler sonography was used to differentiate between vascular and biliary structures.

Results. Laparoscopic ultrasound ensured a safe plane of dissection and no biliary or vascular complications were observed. Stent insertion into the common bile duct before the operation undoubtedly made the identification of anatomical structures easier. Conversion to an open procedure was deemed necessary in only 6 patients (4.8\%).

Conclusions. Laparoscopic ultrasound facilitates the successful performance of LCs. It can be used at any time during the operation; it is noninvasive; and there is no need to use X-rays or contrast dye, or to cannulate the cystic duct. The most important advantage of LUS is that it leads to a lower number of conversions and intraoperative complications by identifying anatomical relationships in the plane of dissection.

Key words: laparoscopic cholecystectomy, bile duct injury, laparoscopic ultrasound 


\section{Introduction}

Bile duct injury (BDI) during open cholecystectomy occurs in $0.1-0.3 \%$ of all cases; in laparoscopic cholecystectomy (LC) this rate ranges from $0.08 \%$ to $0.5 \% .^{1,2}$ According to recent studies, the rates of BDI in LC are approaching the rates in open cholecystectomy, which may reflect better preparation, more sophisticated equipment and more surgical personnel moving beyond the "learning curve". ${ }^{1-5}$ Routine intraoperative cholangiography (IOC) during LC remains a controversial issue and a growing number of surgeons are abandoning it. ${ }^{6}$ Preoperative magnetic resonance cholangiopancreatography can be used to detect choledocholithiasis that can be cleared with endoscopic retrograde cholangiopancreatography (ERCP). If choledocholithiasis is detected after a cholecystectomy, ERCP is performed postoperatively. ${ }^{6}$ Additionally, most occult bile duct stones detected with routine IOC are clinically insignificant. Bile duct injury is not $100 \%$ preventable, and IOC does not provide any information about vascular abnormalities. ${ }^{7}$ Therefore, there is a very strong need for a method that will reduce the number of complications without any additional risks for the patient and the operating team. Laparoscopic ultrasound (LUS) appears to be a very effective tool, especially in difficult cases when the inflammatory process changes anatomical relationships, which can lead to disastrous complications if structures are not identified correctly.

\section{Material and methods}

The study group consisted of 126 patients (80 women and 46 men) operated on between January 2014 and February 2016. Written informed consent was obtained from all the participants, and all the procedures performed in this study involving human participants were in accordance with the ethical standards of the 1964. Helsinki Declaration and its later amendments. The indication for the surgery was symptomatic cholecystolithiasis. The patients selected for the study had difficult intraoperative anatomical conditions due to chronic cholecystitis, previous upper abdominal surgery or biliary pancreatitis (without surgery) in the past (Table 1,2).

The exclusion criteria for the study were acute cholecystitis, acute pancreatitis, gallbladder cancer, and any abdominal operations or acute abdominal conditions within the previous 12 months. The operations were performed on an elective basis by 2 surgeons experienced in LC (>200 LC) and LUS (>100 examinations). We used a Toshiba PEF-704 LA laparoscopic probe (frequency 7.0 MHz) and the Toshiba NemioMX SSA-590A diagnostic ultrasound system (both from Toshiba Corp., Tokyo, Japan). Intraoperative LUS was performed in every patient. The trocar sites were identical to those in standard LC (we used $25-\mathrm{mm}$ trocars and 2 10-millimeter trocars). The ultrasound probe was inserted through the epigastrical (transverse
Table 1. Characteristics of the study group

\begin{tabular}{|l|c|}
\hline Total number of patients & 126 \\
\hline Women/men & $80(63.5 \%) / 46(36.5 \%)$ \\
\hline $\begin{array}{l}\text { Oiliary pancreatitis in the past } \\
\text { ulcer }\end{array}$ & $23(18 \%)$ \\
\hline $\begin{array}{l}\text { Right hemicolectomy for perforation of gastric or duodenal } \\
\text { Chronic inflammation of gallbladder }\end{array}$ & $10(8 \%)$ \\
\hline $\begin{array}{l}\text { Number of conversions } \\
\text { Biliary and/or vascular complications }\end{array}$ & $12(9.5 \%)$ \\
\hline $\begin{array}{l}\text { Stent insertion to the common bile duct before } \\
\text { operation }\end{array}$ & $6(4.8 \%)$ \\
\hline
\end{tabular}

Table 2. Duration of laparoscopic ultrasound and operation

\begin{tabular}{|l|c|}
\hline Time of laparoscopic ultrasound [min] & $5.5(4-9.5)$ \\
\hline $\begin{array}{l}\text { Time of operation [min] - from skin incision till } \\
\text { skin closure }\end{array}$ & $72(53-130)$ \\
\hline
\end{tabular}

view) or umbilical trocar (longitudinal view). The duplex Doppler function facilitated differentiation between vascular and avascular structures. The LUS examination was performed after initial preparation in Calot's triangle and after shifting the gallbladder to the right. The first structure visualized was the "Mickey Mouse sign" - a characteristic configuration of the bile duct, the proper hepatic artery and the portal vein that resembles the head of Mickey Mouse from Walt Disney movies (Fig. 1A,B). We moved up and down from this point and delineated the anatomy (Fig. 2A-D). Preoperative bile duct stenting (due to massive choledocholithiasis and/or narrowing of the bile duct) made ultrasound interpretation easier, because stents are very easy to identify using ultrasound (Fig. 3A-C, Fig. 4A-C). In our practice, we leave stents throughout the surgery and remove them during a subsequent hospitalization.

Our department is the regional center for ERCP and patients who are qualified for LC after ERCP frequently choose our department, which is the reason for the very high percentage $(25.4 \%)$ of patients in the study group with stents in the bile duct. During an LC, we cannot touch the tissues and palpate the stents in the bile duct (they are also invisible from the outside), but we can quite easily find them using LUS. We did not observe any complications in the study group associated with the insertion or removal of stents, but it is not a standard procedure before every LC in our department. The indications for inserting stents are massive choledocholithiasis and/or narrowing of the bile duct.

\section{Results}

Conversion to an open procedure was performed in 6 patients (4.8\%): 2 men ( 1 after acute biliary pancreatitis and the other cancer one with chronic cholecystitis, neither 


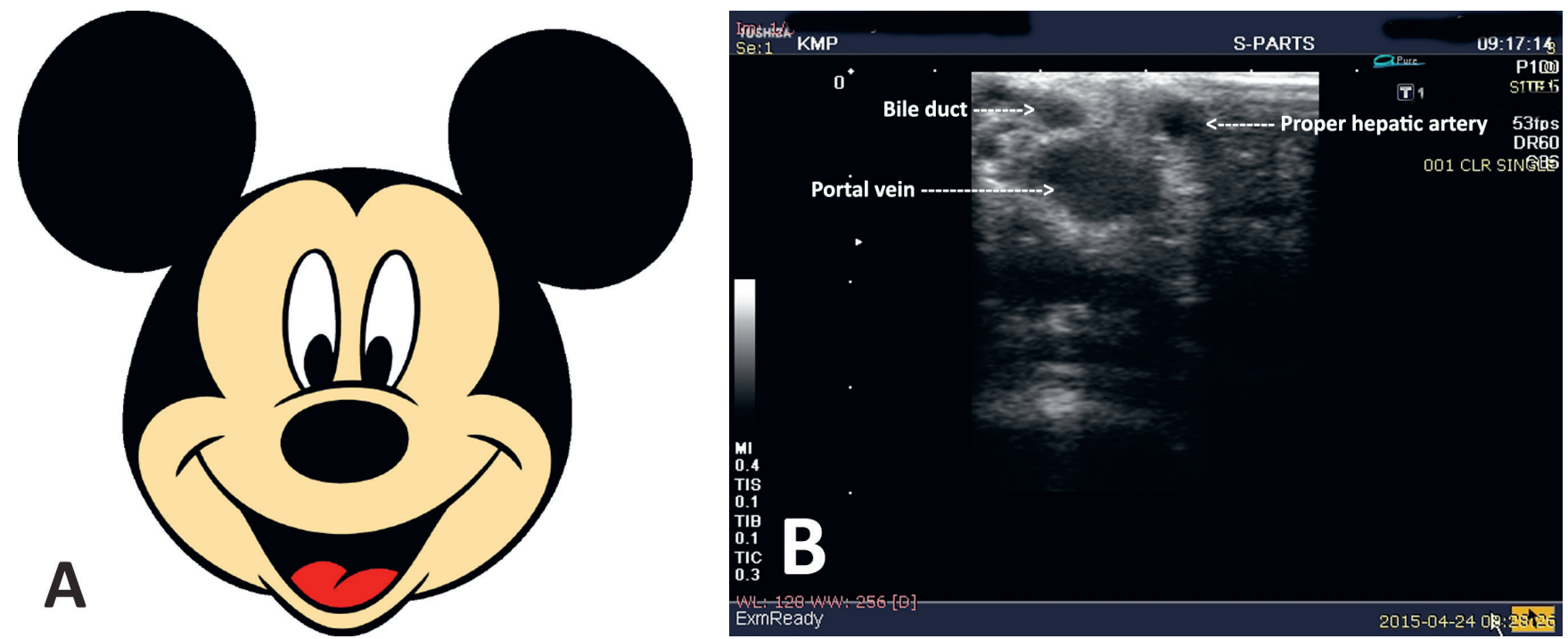

Fig. 1. A - Mickey Mouse; B - the characteristic configuration of the bile duct, the proper hepatic artery and the portal vein in the hepatoduodenal ligament known as the "Mickey Mouse sign"
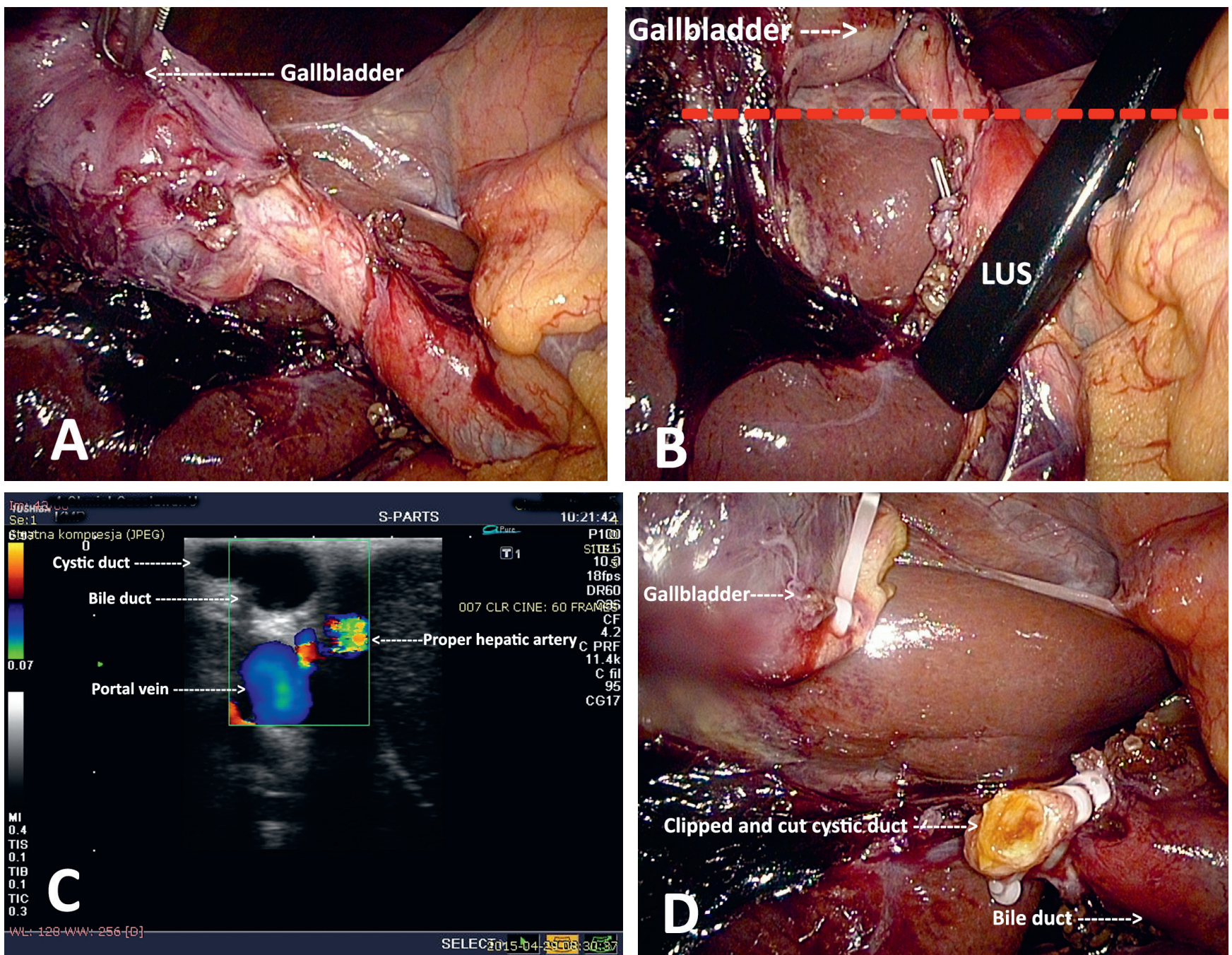

Fig. 2. A - short and wide connection of the cystic duct with the bile duct; chronic cholecystitis; B - LUS defines the correct level of clipping (red line); C - LUS with duplex Doppler function visualizes the connection of the cystic duct with the bile duct, the proper hepatic artery and the portal vein; D - safe clipping and cutting of the cystic duct without narrowing the bile duct 

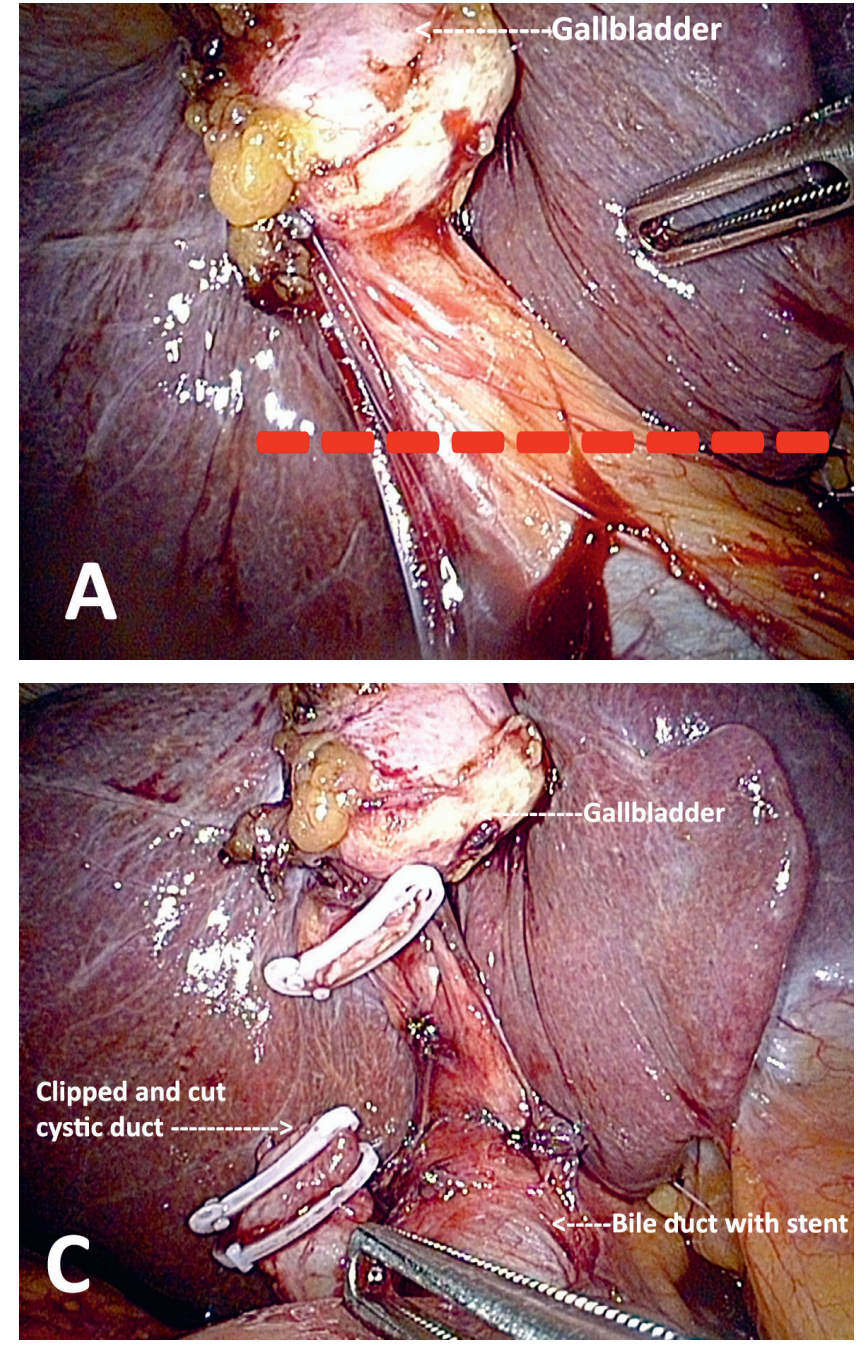

had stents in the biliary tract) and 4 women ( 2 after acute biliary pancreatitis and 2 with chronic cholecystitis, also without stents in the biliary tract). The conversions were due to technical problems: because chronic inflammation and hard infiltrations in the region of the hepatoduodenal ligament prevented safe laparoscopic preparation, open cholecystectomies were performed. The conversions were not caused by bleeding from injured vessels in the hepatoduodenal ligament or BDI. We completed LUS in 100\% of our patients and did not have to perform IOC. To us, successful LUS meant a safe plane of dissection and no biliary and vascular complications; we do not treat conversion as a failure. Stent insertion into the common bile duct (CBD) before surgery undoubtedly made the identification of anatomical structures easier (there were no conversions in this group of patients).

\section{Discussion}

Laparoscopic ultrasound makes the dreams of many surgeons performing LC come true. It minimizes the level of stress associated with clipping and cutting the cystic

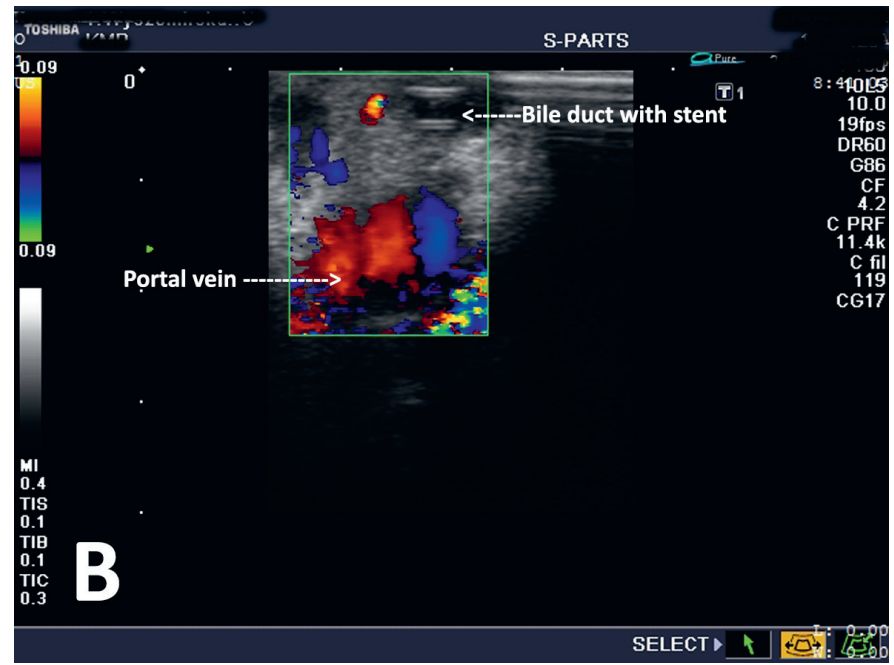

Fig. 3. A - fibrotic and shrunken gallbladder with chronic inflammation; the red line marks the correct level of clipping; B - stent insertion into the bile duct before laparoscopic cholecystectomy $(\mathrm{LC})$ makes ultrasound interpretation easier; $\mathrm{C}$ - the cystic duct is safely clipped and cut without narrowing or injuring the bile duct

duct and the cystic artery. A major advantage of this method is that it is noninvasive and can be performed at any time during the operation. Laparoscopic ultrasound enables us to differentiate between vascular and avascular structures by duplex Doppler; cannulation of the cystic duct and exposure to radiation and contrast dye is avoided; it can be performed during pregnancy; it is possible to visualize the pancreatic head and possible pathology in this localization. ${ }^{8}$ The disadvantages of LUS are undoubtedly the long learning curve, the need for special equipment and limitations to the visualization of the bile ducts. ${ }^{8}$ Intraoperative cholangiography allows vizualization of the entire biliary tree and finding abnormal anatomic configurations. In comparison to IOC, LUS is less time-consuming and cheaper, but it has comparable sensitivity and specificity in terms of confirmations of anatomical relationships and the presence of bile duct stones. ${ }^{8}$ In a study by Teefey et al. IOC confirmed aberrant anatomy in $14 \%$ of the patients not found in LUS $^{9}$; in another study LUS detected $82 \%$ of anomalies found on IOC. ${ }^{10}$ On the other hand, Wysocki's study found that sound surgical judgment combined with a critical view of safety (CVS) are vital to preventing BDI, and routine IOC does not fully protect against major BDI. ${ }^{11}$ If the bile duct is mistaken for the cystic duct, IOC can itself lead to an incision causing Strasberg type D BDI. ${ }^{11-14}$ A study by Biffl et al. showed that the routine use of LUS was associated with a lack of complications from bile ducts, while $2.5 \%$ of the patients who underwent surgery without LUS had bile complications; there were also fewer conversions in the LUS group. ${ }^{15}$ Machi et al. reported that in 1,381 LCs with routine use of LUS, no BDI was observed and the conversion rate was lower (6\%); additional IOC was necessary in only $2 \%$ of the cases. ${ }^{16} \mathrm{We}$ consider the key to a safe LC 

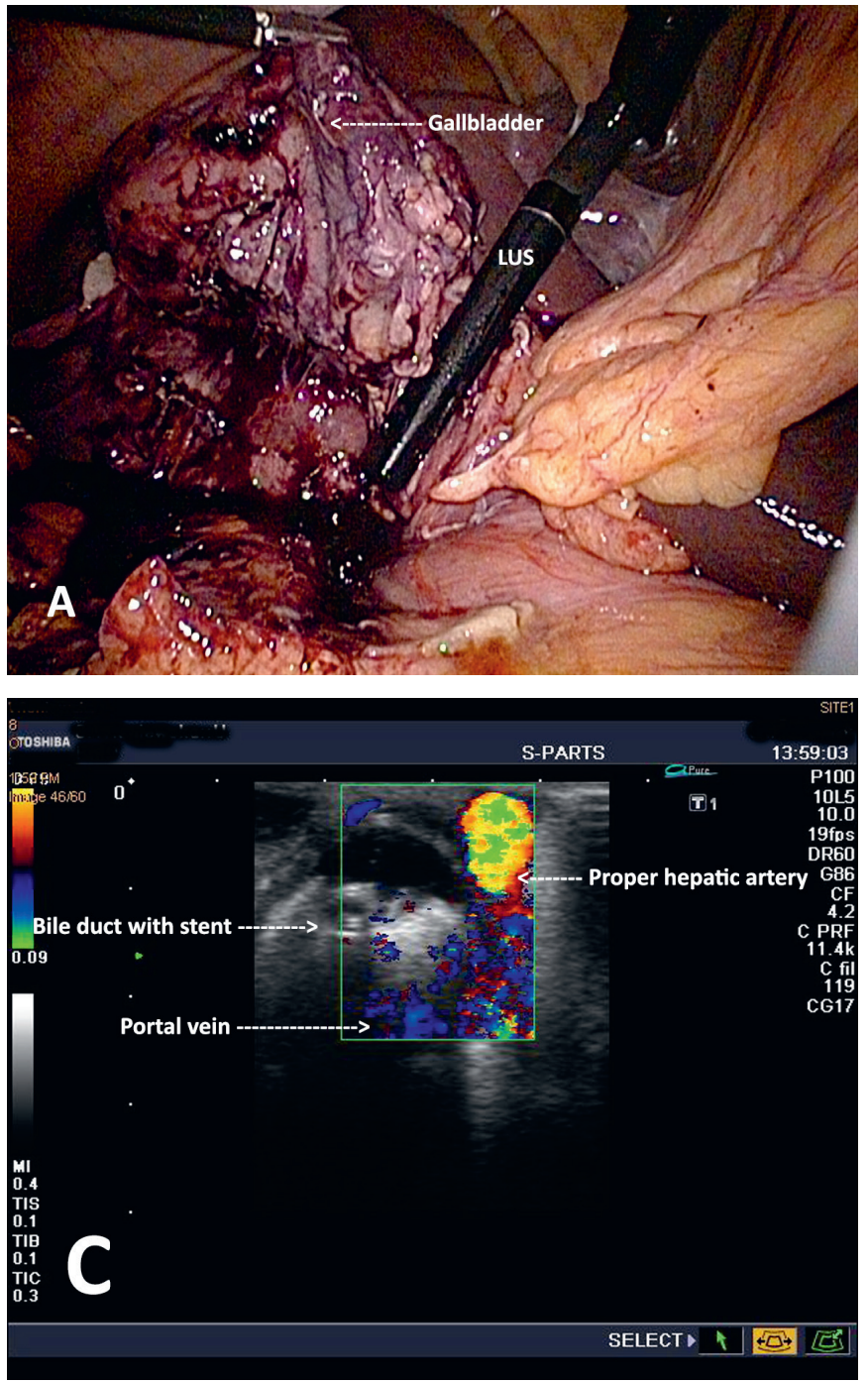

is confirmation of the right plane of dissection. We agree with other authors that as a noninvasive, safe, less costly and faster method, LUS should be the primary imaging method during LC, whereas IOC should be used only when any doubts exist. ${ }^{7,17}$ Our success rate with LUS was $100 \%$ (a safe plane of dissection, no biliary or vascular complications), while the success rate for other authors varied between 95-100\% with LUS and 89-97\% with IOC (albeit other authors' definition of "success" was not clear). ${ }^{18}$

The conversion rate from LC to open cholecystectomy varies from $2.6 \%$ to $11.9 \%$. Factors associated with a need for conversion are the degree of inflammation, previous upper abdominal surgery, acute cholecystitis, advanced age, male gender, dense adhesions, and obscure anatomy in Calot's triangle. ${ }^{19,20}$ Laparoscopic ultrasound can diminish the rate of conversions without additional risk for the patient by providing exact navigation in the operating field: the decision of whether or not to convert to open surgery is based on visual monitoring of the vessels and the CBD, not on leaking bile and a hole in the portal vein or the hepatic artery. The question of protective bile duct stenting before surgery remains open; in our study

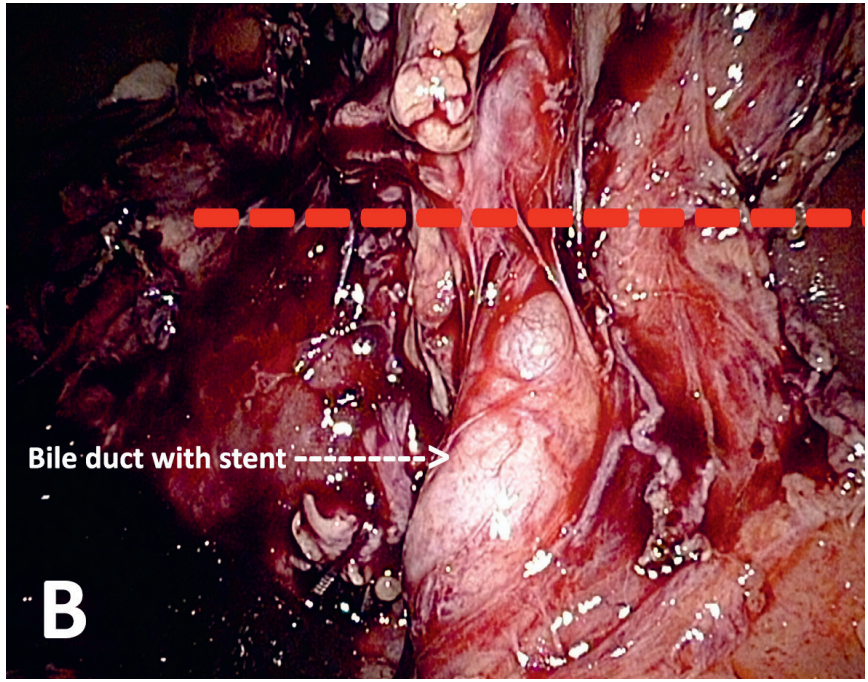

Fig. 4. A - chronic cholecystitis; laparoscopic ultrasound (LUS); B - chronic inflammation in the neck of the gallbladder; further dissection, clipping and cutting under the red line would lead to severe biliary and vascular complications; $\mathrm{C}$ - the stent in the bile duct is visible in the ultrasound. Laparoscopic ultrasound with duplex Doppler function defines the correct level of further dissection

it helped to delineate anatomy, and there were no conversions in the group of patients given stents prior to the LC.

Of course, we cannot forget that apart from methods that are the effect of technological development, according to Strasberg's 1995 recommendations, a very important part of LC is a correct surgical technique and obtaining CVS. We have to separate the lower third of the gallbladder from the liver, clean the region of Calot's triangle (we should see the V segment through the established window); the cystic duct and the cystic artery should be the only visible ductal structures left between the gallbladder and the CBD. Sometimes the cystic artery is cut with electrocautery close to the gallbladder, in which case only the cystic duct is visible. If we cannot obtain CVS, we should perform an open cholecystectomy or use additional methods of visualization. ${ }^{21,22}$ If we have any doubts, we should use LUS and sigh with relief that we have not followed a misleading route directly to severe complications.

To conclude, LUS as a method of intraoperative guidance during difficult LC helps the surgeon navigate in a hostile environment and allows the procedure to be successfully performed. It enables the surgeon to be certain of the structures being identified, clipped and cut intraoperatively, which is of utmost importance during a difficult LC.

\section{References}

1. Tropea A, Pagano D, Biondi A, Spada M, Gruttadauria S. Treatment of the iatrogenic lesion of the biliary tree secondary to laparoscopic cholecystectomy: A single center experience. Updates Surg. 2016; 68(2):143-148.

2. Halbert C, Pagkratis S, Yang J, et al. Beyond the learning curve: Incidence of bile duct injuries following laparoscopic cholecystectomy normalize to open in the modern era. Surg Endosc. 2016;30(6): 2239-2243. 
3. Gentileschi P, Di Paola M, Catarci M, et al. Bile duct injuries during laparoscopic cholecystectomy: A 1994-2001 audit on 13,718 operations in the area of Rome. Surg Endosc. 2004;18(2):232-236.

4. Nuzzo G, Giuliante F, Giovannini I, et al. Bile duct injury during laparoscopic cholecystectomy: Results of an Italian national survey on 56591 cholecystectomies. Arch Surg. 2005;140(10):986-992.

5. Hamad MA, Nada AA, Abdel-Atty MY, Kawashti AS. Major biliary complications in 2,714 cases of laparoscopic cholecystectomy without intraoperative cholangiography: A multicenter retrospective study. Surg Endosc. 2011;25(12):3747-3751.

6. Aziz O, Ashrafian $\mathrm{H}$, Jones $\mathrm{C}$, et al. Laparoscopic ultrasonography versus intra-operative cholangiogram for the detection of common bile duct stones during laparoscopic cholecystectomy: A meta-analysis of diagnostic accuracy. Int J Surg. 2014;12(7):712-719.

7. Machi J, Oishi AJ, Tajiri T, Murayama KM, Furumoto NL, Oishi RH. Routine laparoscopic ultrasound can significantly reduce the need for selective intraoperative cholangiography during cholecystectomy. Surg Endosc. 2007;21(2):270-274.

8. Yao CC, Huang SM, Lin CC, et al. Assessment of common bile duct using laparoscopic ultrasound during laparoscopic cholecystectomy. Surg Laparosc Endosc Percutan Tech. 2009;19(4):317-320.

9. Teefey SA, Soper NJ, Middleton WD, et al. Imaging of the common bile duct during laparoscopic cholecystectomy: Sonography versus videofluoroscopic cholangiography. AJR Am J Roentgenol. 1995;165(4): 847-851.

10. Röthlin $M$, Largiadèr $F$. The anatomy of the hepatoduodenal ligament in laparoscopic sonography. Surg Endosc. 1994;8(3):173-180.

11. Wysocki AP. Population-based studies should not be used to justify a policy of routine cholangiography to prevent major bile duct injury during laparoscopic cholecystectomy. World J Surg. 2016;41(1): 82-89.

12. Beksac K, Turhan N, Karaagaoglu E, Abbasoglu O. Risk factors for conversion of laparoscopic cholecystectomy to open surgery: A new predictive statistical model. J Laparoendosc Adv Surg Tech A. 2016;26(9): 693-696.
13. Sutcliffe RP, Hollyman M, Hodson J, Bonney G, Vohra RS, Griffiths EA; CholeS study group, West Midlands Research Collaborative. Preoperative risk factors for conversion from laparoscopic to open cholecystectomy: A validated risk score derived from a prospective UK database of 8820 patients. HPB (Oxford). 2016;1365-182X(16):31837-31838.

14. Le VH, Smith DE, Johnson BL. Conversion of laparoscopic to open cholecystectomy in the current era of laparoscopic surgery. Am Surg. 2012;78(12):1392-1395.

15. Biffl WL, Moore EE, Offner PJ, Franciose RJ, Burch JM. Routine intraoperative laparoscopic ultrasonography with selective cholangiography reduces bile duct complications during laparoscopic cholecystectomy. J Am Coll Surg. 2001;193(3):272-280.

16. Machi J, Johnson JO, Deziel DJ. The routine use of laparoscopic ultrasound decreases bile duct injury: A multicenter study. Surg Endosc. 2009;23(2):384-388.

17. Jamal KN, Smith H, Ratnasingham K, Siddiqui MR, McLachlan G, Belgaumkar AP. Meta-analysis of the diagnostic accuracy of laparoscopic ultrasonography and intraoperative cholangiography in detection of common bile duct stones. Ann R Coll Surg Engl. 2016;98(4): 244-249.

18. Perry KA, Myers JA, Deziel DJ. Laparoscopic ultrasound as the primary method for bile duct imaging during cholecystectomy. Surg Endosc. 2008;22(1):208-213.

19. Malik AM. Difficult laparoscopic cholecystectomies. Is conversion a sensible option? J Pak Med Assoc. 2015;5(7):698-700.

20. Lee NW, Collins J, Britt R. Evaluation of preoperative risk factors for converting laparoscopic to open cholecystectomy. Am Surg. 2012; 78(8):831-833.

21. Buddingh KT, Nieuwenhuijs VB, van Buuren L, Hulscher JB, de Jong JS, van Dam GM. Intraoperative assessment of biliary anatomy for prevention of bile duct injury: A review of current and future patient safety interventions. Surg Endosc. 2011;25(8):2449-2461.

22. Strasberg SM, Hertl M, Soper NJ. An analysis of the problem of biliary injury during laparoscopic cholecystectomy. Am Coll Surg. 1995; 180(1):101-125. 